\title{
Analysis of Association Between Various Clinical Forms of Tuberculosis and SLC11A1 and IFNG Gene Polymorphisms in Individuals of Yakut Ethnicity
}

\author{
M.V. Egorova1 ${ }^{1}$, L.I. Mordovskaya ${ }^{1,2 *}$, T.M. Klimova ${ }^{2}$, V.V. Gerasimova ${ }^{2,3}$ and A.R. \\ Ermolaev $^{3}$ \\ ${ }^{1}$ Phthisiatry Research-Practice Center name by.E.N. Andreev, 93, P. Alexeev str., Yakutsk \\ ${ }^{2}$ Medical Institute, M.K. Ammosov North-Eastern Federal University name by M.K. Ammosov. 27, Oyunskogo str, \\ Yakutsk. \\ ${ }^{3}$ Blood Transfusion Center, 85. P. Alexeev str., Yakutsk \\ *Corresponding author.Email: mordovskaya.li@s-vfu.ru
}

\begin{abstract}
172 Yakut patients with confirmed tuberculosis (TB) were included to the study, exploring the possible association between polymorphisms in SLC11A1 and IFNG genes and various clinical forms of TB in patients of Yakut ethnicity. Distributions of single nucleotide polymorphisms (SNPs) were analyzed in Yakut patients, in SLC11A1 gene Asp543Asn locus, in IFNG gene T6000A and T8360C loci, in relation to disease extent, clinical progression pattern, culture test results, presence of necrotic cavities. No association was detected between polymorphisms in genes SLC11A1 (rs17235409) or IFNG (rs2430561, rs2069718) and disease localization, clinical form, culture test result, or clinical progression pattern in patients with TB.
\end{abstract}

Keywords: tuberculosis, SLC11A1 and IFNG gene polymorphisms, Yakut ethnicity

\section{INTRODUCTION}

Tuberculosis (TB) is known as one of the most widespread infectious diseases in the world. Based on the WHO report, 10 million were diagnosed and 1.5 million people across the globe died of TB in 2018. According to current estimations, nearly one third of the world population is infected with Mycobacterium tuberculosis (MTB), but only $10 \%$ develop clinical disease. This fact suggests that powerful mechanisms of resistance to TB infection are at work in humans. In other words, resistance to TB is apparently controlled by resistance and susceptibility genes [2]. Determination of genetic markers for susceptibility to pulmonary TB disease is the prerequisite for well-targeted population distribution to risk groups for $\mathrm{TB}$, and more importantly, these risk groups should be defined specifically for each region [3, 4]. Study of genetic determinants for this dreadful infection provides the ways to understand underlying mechanisms for $\mathrm{TB}$ immunity and disease, as well as, to harness genotyping methods for the purposes of better TB diagnosis and treatment [1].

Problem statement: The advent of molecular genetic methods has resulted in deciphering a great many of mutations in cytokine system, and establishing their associations with infectious diseases, including TB. Population studies have shown polymorphisms in various genes to be associated with TB. For example, in a study of $+874 \mathrm{~A} / \mathrm{T}$ mutation in IFNG gene in the Chinese population, mutations were found reliably more often in allele A, in TB group [10]. A study in Pakistani population [5] compared healthy controls with patients diagnosed with various clinical forms of TB, and IFNG gene $+874 \mathrm{~T} / \mathrm{T}$ mutation was shown to be associated with minor lesions of pulmonary TB, while +874T/A mutation was associated with severe pulmonary $\mathrm{TB}$ or extrapulmonary TB. Study in Rijeka, Croatia, among 253 hospitalized patients and 519 controls analyzed 
association between IFNG gene polymorphisms ( $\mathrm{T}$ $+874 \mathrm{~A}, \mathrm{G}+2109 \mathrm{~A}$ ) and predisposition to TB. Genotypes T/T +874 (possibly, high IFNG producers) and $+874 \mathrm{~A} / \mathrm{A}$ (possibly, low IFNG producers) were observed to associate with AFB-positive and negative forms of TB. These findings were confirmed by haplotypes $\mathrm{T}+874 \mathrm{~A}$ and $\mathrm{G}+2109 \mathrm{~A}$. The patients showed higher frequencies of genotypes missing $\mathrm{T}$ in +874 (AA/AA; AA/AG; AG/AG), among AFB/culturepositive patients, compared to negative patients. Seemingly, IFNG gene polymorphism is associated with disease severity, but not susceptibility to $\mathrm{TB}$, in a Croatian population $[7,11]$.

Influence of IFNG gene polymorphisms on treatment response to TB in 77 Japanese patients with culture-confirmed drug-sensitive TB showed no significant differences between subjects with AA genotype and those with the other genotypes which seemed to affect the clinical course of TB treatment. AA genotype was observed to be associated with slower culture conversion, compared to other genotypes. The study showed that the lower IFNG-producing +874 AA genotype was related to the delay to sputum culture conversion compared with the other genotypes, suggesting a quantitative effect of IFNG on TB treatment. This delay in mycobacterial culture conversion during TB treatment has another implication. Patients with sputum smears negative for acid-fast bacilli can still transmit M.tuberculosis theoretically and empirically, while they are less infectious than those with positive smears. Because it is not practical to isolate every patient with TB until culture conversion is confirmed in order to prevent transmission, clinical evaluation of the risk of transmission was important for public health. The AA genotype was strongly and independently predictive of a lower likelihood of sputum conversion. Indeed, 4 of 56 patients with the IFNG +874 AA genotypes had not achieved the culture negativity at 3 month. These results indicated that this polymorphism is one of the important predictive factors for the duration of isolation from the viewpoint of public health as well as the individual clinical course of TB treatment. [9].

Frequencies of IFNG gene $+874 \mathrm{~T} / \mathrm{A}$ polymorphisms in healthy individuals versus pulmonary TB patients were studied in Zahedan, Southeast Iran. Based on findings, + 874T/A SNP in intronic sequence of IFNG gene (rs61923114) could be related to susceptibility to TB in the population of Iran. Genotype $+874 \mathrm{AA}$ and $+874 \mathrm{~A}$ allele were more frequent in patients diagnosed with $\mathrm{TB}$, then in those with non-TB diagnoses. The results were consistent with those from other studies conducted among other populations, and were suggesting association between IFNG gene +874 TA polymorphism and susceptibility to TB [8].

Despite numerous studies of different polymorphisms of the genes governing TB infection, there are still gaps in knowledge. It is not clear, which mutations in which of the genes are critically important. Possibly, what plays role is not alleles alone, but their interactions. Studies continue, as the rest of polymorphisms need to be further explored, with larger patient groups and more groups for comparisons.

\section{RESEARCH QUESTIONS}

The study comprised 172 Yakut patients diagnosed with TB, who had been hospitalized in Phthisiatry Research-Practice Center from 2016 to 2018. All patients gave informed consent to undergo genetic testing.

Mean patient age was $34.7(17.9)$ years. Majority of them were rural residents $(108 ; 62.8 \%)$, and smaller proportions were urban $(64 ; 37.2 \%)$, socially marginalized $(48 ; 30 \%)$, unemployed $(85 ; 49.4 \%)$, students $(45 ; 26.1 \%)$. Exposure to TB was traced in 81 $(47.0 \%)$ cases, of them $50.6 \%$ cases were family contacts.

Genomic DNA was extracted from blood using DNA-Extran kit (Sintol); DNA genotyping was performed using CFX96TM Real-Time PCR system (Biorad). Polymorphism detection kits for the following SNPs were used: Asp543Asn in SLC11A1 gene (rs17235409), T6000A in IFNG gene (rs2430561), T8360C in IFNG gene (rs2069718) (Sintol).

Statistical analysis was performed using IBM SPSS Statistics 22 software, and Gene Expert online genetic calculation tool (http://gen-exp.ru/calculatoror.php). Allelic and genotypic frequencies of polymorphisms in genes SLC11A1 (rs17235409) and IFNG (rs2430561 and rs2069718) were compared between cases and controls based on Pearson's chi-squared test, and odds ratios with $95 \%$ confidence interval (CI). Ratios of genotype frequencies to allelic variants were tested against Hardy-Weinberg equilibrium.

The aim of this paper is to study if various clinical forms of $\mathrm{TB}$ in Yakut population are associated with SLC11A1 or IFNG gene polymorphisms.

Methods: conventional laboratory and clinical study methods.

The study comprised $105(61.0 \%)$ men, and 67 $(39.0 \%)$ women. Mean patient age was 34.7 (17.9). The majority of patients were rural residents $(108 ; 62.8 \%)$; number of urban residents was 64 (37.2\%); 48 (30\%) patients were socially-deprived; 85 (49.4\%) were unemployed; $45(26.1 \%)$ were students. Exposure to active TB case was traced in $81(47.0 \%)$ patients in case group, of them, $50.6 \%$ were family contacts.

Clinical TB forms in TB case group are shown in Table 1. 
Table 1. Clinical TB forms distribution

\begin{tabular}{|l|l|l|}
\hline Diagnosis & N & $\%$ \\
\hline Disseminated TB & 25 & 14.5 \\
\hline Infiltrative TB & 83 & 48.3 \\
\hline Cavitary TB & 2 & 1.2 \\
\hline Caseous pneumonia & 2 & 1.2 \\
\hline Focal TB & 20 & 11.6 \\
\hline Primary complex & 7 & 4.0 \\
\hline TB of intrathoracic lymph nodes & 11 & 6.4 \\
\hline TB of bronchi & 2 & 1.2 \\
\hline Tuberculous lymphadenitis & 1 & 0.6 \\
\hline Tuberculoma & 5 & 2.9 \\
\hline Fibrotic cavitary TB & 11 & 6.4 \\
\hline Cirrhotic TB & 3 & 1.7 \\
\hline Total & 172 & 100.0 \\
\hline
\end{tabular}

We analyzed distributions of Asp543Asn (SLC11A1 gene), T6000A and T8360C (IFNG gene) SNPs in Yakut patients with TB in relation to: disease extent, clinical progression pattern, culture test result, presence of necrotic cavities.

Absolute and relative frequencies of genotypes and alleles in patients with $\mathrm{TB}$, depending on disease localization, clinical form, culture test results, clinical progression patterns, are shown in tables 2-8.

Respectively $59 \%$ of TB cases and $63 \%$ of healthy controls were homozygous carriers of TT genotype. The rare AA genotype was seen in 5.8 and $5.4 \%$, respectively. No statistically significant differences were established in genotype distributions between TB cases and healthy controls $(\mathrm{p}=0.72)$.

Differences in major, heterozygous, or minor genotype frequencies were statistically negligible between TB cases and controls $(p=0.89)$. Proportion of individuals carrying minor genotype was small.

Table 2. Distribution of allele and genotype frequencies for SLC11A1 Asp 543 Asn (rs17235409) polymorphism in TB patients and healthy individuals

\begin{tabular}{|c|c|c|c|c|c|c|}
\hline \multirow[t]{2}{*}{ rs17235409 } & TB cases & Controls & \multirow[t]{2}{*}{$x^{2}$} & \multirow[t]{2}{*}{$P$} & \multicolumn{2}{|l|}{ OR } \\
\hline & $n=172$ & $n=367$ & & & $\overline{\text { Value }}$ & $95 \% \mathrm{Cl}$ \\
\hline \multicolumn{7}{|l|}{ Alleles } \\
\hline $\mathrm{G}$ & 82.0 & 79.2 & \multirow[t]{2}{*}{1.17} & \multirow[t]{2}{*}{0.28} & 1.20 & $0.86-1.66$ \\
\hline $\mathrm{A}$ & 18.0 & 20.8 & & & 0.83 & $0.60-1.16$ \\
\hline \multicolumn{7}{|l|}{ Genotypes } \\
\hline GG & 66.9 & 63.8 & \multirow[t]{2}{*}{1.61} & \multirow[t]{2}{*}{0.45} & 1.16 & $0.79-1.70$ \\
\hline GA & 30.2 & 31.3 & & & 0.95 & $0.64-1.41$ \\
\hline
\end{tabular}

Table 3. Distribution of allele and genotype frequencies for IFN- $\gamma$ T6000A (rs2430561) polymorphism between TB patients and healthy individuals

\begin{tabular}{|c|c|c|c|c|c|c|}
\hline \multirow{2}{*}{ rs2430561 } & \multirow{2}{*}{\begin{tabular}{|l} 
TB cases \\
$n=172$
\end{tabular}} & \multirow{2}{*}{$\frac{\text { Controls }}{n=367}$} & \multirow{2}{*}{$x^{2}$} & \multirow[t]{2}{*}{$P$} & \multicolumn{2}{|l|}{$\overline{O R}$} \\
\hline & & & & & Value & $95 \% \mathrm{Cl}$ \\
\hline \multicolumn{7}{|l|}{ Alleles } \\
\hline$T$ & 76.7 & 78.7 & \multirow[t]{2}{*}{0.55} & \multirow[t]{2}{*}{0.46} & 0.89 & $0.66-1.21$ \\
\hline$A$ & 23.3 & 21.3 & & & 1.12 & $0.83-1.53$ \\
\hline \multicolumn{7}{|l|}{ Genotypes } \\
\hline TT & 59.3 & 62.9 & \multirow[t]{3}{*}{0.66} & \multirow[t]{3}{*}{0.72} & 0.86 & $0.59-1.24$ \\
\hline TA & 34.9 & 31.6 & & & 1.16 & $0.79-1.70$ \\
\hline AA & 5.8 & 5.4 & & & 1.07 & $0.49-2.34$ \\
\hline
\end{tabular}


Table 4. Distribution of allele and genotype frequencies for IFN- $\gamma$ T8360C (rs2069718) polymorphism between TB patients and healthy individuals

\begin{tabular}{|c|c|c|c|c|c|c|}
\hline \multirow[t]{2}{*}{ rs2069718 } & \multirow{2}{*}{\begin{tabular}{|l|} 
TB cases \\
$n=172$ \\
\end{tabular}} & \multirow{2}{*}{\begin{tabular}{|l|} 
Controls \\
$n=367$ \\
\end{tabular}} & \multirow{2}{*}{$x^{2}$} & \multirow[t]{2}{*}{$P$} & \multicolumn{2}{|l|}{$O R$} \\
\hline & & & & & Value & $95 \% \mathrm{Cl}$ \\
\hline \multicolumn{7}{|l|}{ Alleles } \\
\hline $\mathrm{T}$ & 80.5 & 81.1 & \multirow[t]{2}{*}{0.04} & \multirow[t]{2}{*}{0.83} & 0.97 & $0.70-1.34$ \\
\hline C & 19.5 & 18.9 & & & 1.04 & $0.75-1.43$ \\
\hline \multicolumn{7}{|l|}{ Genotypes } \\
\hline TT & 64.5 & 64.9 & \multirow[t]{3}{*}{0.24} & \multirow[t]{3}{*}{0.89} & 0.99 & $0.68-1.44$ \\
\hline $\mathrm{TC}$ & 32.0 & 32.4 & & & 0.98 & $0.66-1.44$ \\
\hline $\mathrm{CC}$ & 3.5 & 2.7 & & & 1.29 & $0.46-3.61$ \\
\hline
\end{tabular}

Table 5. SLC11A1 and IFNG gene polymorphisms in patients with $\mathrm{TB}$, in relation to disease localization, $\mathrm{n}(\%)$

\begin{tabular}{|c|c|c|c|c|}
\hline Genotype & $\begin{array}{l}\text { Pulmonary } \\
\text { TB } n=169\end{array}$ & $\begin{array}{l}\text { Extrapulmonary } \\
\text { TB } n=3\end{array}$ & $x^{2}$ & $p$ \\
\hline \multicolumn{5}{|c|}{ Asp543Asn SNP in SLC11A1 gene } \\
\hline $\mathrm{AA}$ & $5(3)$ & $0(0)$ & \multirow[t]{3}{*}{0.098} & \multirow[t]{3}{*}{0.952} \\
\hline $\mathrm{GA}$ & $51(30.2)$ & $1(33.3)$ & & \\
\hline GG & $113(66.9)$ & $2(66.7)$ & & \\
\hline \multicolumn{5}{|c|}{ T6000A SNP in IFNG gene } \\
\hline AA & $9(5.3)$ & 1 (33.3) & \multirow[t]{3}{*}{4.326} & \multirow[t]{3}{*}{0.115} \\
\hline TA & $59(34.9)$ & $1(33.3)$ & & \\
\hline TT & $101(59.8)$ & $1(33.3)$ & & \\
\hline \multicolumn{5}{|c|}{ T8360C SNP in IFNG gene } \\
\hline $\mathrm{CC}$ & $6(3.6)$ & $0(0)$ & \multirow[t]{3}{*}{0.110} & \multirow[t]{3}{*}{0.946} \\
\hline TC & $54(32)$ & $\begin{array}{l}1(33.3) \\
\end{array}$ & & \\
\hline TT & $109(64.5)$ & $2(66.7)$ & & \\
\hline
\end{tabular}

Table 6. SLC11A1 and IFNG gene polymorphisms in patients to primary or post-primary $\mathrm{TB}, \mathrm{n}(\%)$

\begin{tabular}{|c|c|c|c|c|}
\hline Genotype & $\begin{array}{l}\text { Primary TB, } \\
n=21\end{array}$ & $\begin{array}{l}\text { Post-primary } \\
\text { TB, } n=151\end{array}$ & $x^{2}$ & $\mathrm{p}$ \\
\hline \multicolumn{5}{|c|}{ Asp543Asn SNP in SLC11A1 gene } \\
\hline AA & $1(4.8)$ & $4(2.6)$ & \multirow[t]{3}{*}{0.447} & \multirow[t]{3}{*}{0.800} \\
\hline GA & $7(33.3)$ & $45(29.8)$ & & \\
\hline GG & $13(61.9)$ & $102(67.5)$ & & \\
\hline \multicolumn{5}{|c|}{ T6000A SNP in IFNG gene } \\
\hline AA & $2(9.5)$ & $8(5.3)$ & \multirow[t]{3}{*}{0.830} & \multirow[t]{3}{*}{0.660} \\
\hline TA & $8(38.1)$ & $52(34.4)$ & & \\
\hline TT & $11(52.4)$ & $91(60.3)$ & & \\
\hline \multicolumn{5}{|c|}{ T8360C SNP in IFNG gene } \\
\hline $\mathrm{CC}$ & $2(9.5)$ & $4(2.6)$ & \multirow[t]{3}{*}{2.61} & \multirow[t]{3}{*}{0.271} \\
\hline TC & $6(28.6)$ & $49(32.5)$ & & \\
\hline TT & $13(61.9)$ & $98(64.9)$ & & \\
\hline
\end{tabular}

Table 7. SLC11A1 and IFNG gene polymorphisms in relation to chronic or other forms of TB, n ( \%)

\begin{tabular}{|c|c|c|c|c|}
\hline Genotype & $\begin{array}{l}\text { Other forms of } \\
\text { TB, } n=155\end{array}$ & $\begin{array}{l}\text { Chronic TB, } \\
n=17\end{array}$ & $x^{2}$ & p \\
\hline \multicolumn{5}{|c|}{ Asp543Asn SNP in SLC11A1 gene } \\
\hline AA & $4(2.6)$ & $1(5.9)$ & \multirow[t]{3}{*}{1.868} & \multirow[t]{3}{*}{0.393} \\
\hline GA & $45(29)$ & $7(41.2)$ & & \\
\hline GG & $106(68.4)$ & $9(52.9)$ & & \\
\hline \multicolumn{5}{|c|}{ T6000A SNP in IFNG gene } \\
\hline AA & $10(6.5)$ & $0(0)$ & \multirow[t]{3}{*}{2.732} & \multirow[t]{3}{*}{0.255} \\
\hline TA & $56(36.1)$ & $4(23.5)$ & & \\
\hline TT & $89(57.4)$ & $13(76.5)$ & & \\
\hline \multicolumn{5}{|c|}{ T8360C SNP in IFNG gene } \\
\hline $\mathrm{CC}$ & $6(3.9)$ & $0(0)$ & \multirow[t]{3}{*}{2.798} & \multirow[t]{3}{*}{0.247} \\
\hline TC & $52(33.5)$ & $3(17.6)$ & & \\
\hline TT & $97(62.6)$ & $14(82.4)$ & & \\
\hline
\end{tabular}

Table 8. SLC11A1 and IFNG gene polymorphisms in patients with $\mathrm{TB}$, in relation to disease extent, $\mathrm{n}(\%)$

\begin{tabular}{|c|c|c|c|c|}
\hline Genotype & $\begin{array}{l}\text { Localized } \\
\text { TB, } n=126\end{array}$ & $\begin{array}{l}\text { Extensive } \\
\text { TB, } n=46\end{array}$ & $x^{2}$ & $p$ \\
\hline \multicolumn{5}{|c|}{ Asp543Asn SNP in SLC11A1 gene } \\
\hline AA & $4(3.2)$ & $1(2.2)$ & \multirow[t]{3}{*}{0.120} & \multirow[t]{3}{*}{0.942} \\
\hline $\mathrm{GA}$ & $38(30.2)$ & $14(30.4)$ & & \\
\hline GG & $84(66.7)$ & $31(67.4)$ & & \\
\hline \multicolumn{5}{|c|}{ T6000A SNP in IFNG gene } \\
\hline AA & $8(6.3)$ & $2(4.3)$ & \multirow[t]{3}{*}{0.258} & \multirow[t]{3}{*}{0.879} \\
\hline TA & $44(34.9)$ & $16(34.8)$ & & \\
\hline TT & $74(58.7)$ & $28(60.9)$ & & \\
\hline \multicolumn{5}{|c|}{ T8360C SNP in IFNG gene } \\
\hline $\mathrm{CC}$ & $3(2.4)$ & $3(6.5)$ & \multirow[t]{3}{*}{1.932} & \multirow[t]{3}{*}{0.381} \\
\hline $\mathrm{TC}$ & $42(33.3)$ & $13(28.3)$ & & \\
\hline TT & $81(64.3)$ & $30(65.2)$ & & \\
\hline
\end{tabular}

Table 9. SLC11A1 and IFN- $\gamma$ gene polymorphisms in TB patients in relation to presence of MTB in sputum, $n$ $(\%)$

\begin{tabular}{|c|c|c|c|c|}
\hline Genotype & $\begin{array}{l}\text { MTB-, } \\
n=56\end{array}$ & $\begin{array}{l}\text { MTB+, } \\
n=115\end{array}$ & $x^{2}$ & $p$ \\
\hline \multicolumn{5}{|c|}{ Asp543Asn SNP in SLC11A1 gene } \\
\hline AA & $3(5.4)$ & $2(1.7)$ & \multirow[t]{3}{*}{2.468} & \multirow[t]{3}{*}{0.291} \\
\hline GA & $19(33.9)$ & $33(28.7)$ & & \\
\hline GG & $34(60.7)$ & $80(69.6)$ & & \\
\hline \multicolumn{5}{|c|}{ T6000A SNP in IFNG gene } \\
\hline AA & $3(5.4)$ & $7(6.1)$ & \multirow[t]{3}{*}{1.309} & \multirow[t]{3}{*}{0.520} \\
\hline TA & $23(41.1)$ & $37(32.2)$ & & \\
\hline TT & $30(53.6)$ & $71(61.7)$ & & \\
\hline \multicolumn{5}{|c|}{ T8360C SNP in IFNG gene } \\
\hline $\mathrm{CC}$ & $2(3.6)$ & $4(3.5)$ & \multirow[t]{3}{*}{0.496} & \multirow[t]{3}{*}{0.780} \\
\hline $\mathrm{TC}$ & $20(35.7)$ & $35(30.4)$ & & \\
\hline TT & $34(60.7)$ & $76(66.1)$ & & \\
\hline
\end{tabular}

Table 10. SLC11A1 and IFN- $\gamma$ gene polymorphisms in TB patients in relation to the presence of lung tissue necrotic cavitation, $\mathrm{n}(\%)$

\begin{tabular}{|c|c|c|c|c|}
\hline Genotype & $\begin{array}{l}\text { Cavities } \\
\text { present, } \\
n=47\end{array}$ & $\begin{array}{l}\text { Cavities } \\
\text { absent, } \\
n=125\end{array}$ & $x^{2}$ & $\bar{p}$ \\
\hline \multicolumn{5}{|c|}{ Asp543Asn SNP in SLC11A1 gene } \\
\hline $\mathrm{AA}$ & $0(0)$ & $5(4)$ & \multirow[t]{3}{*}{4.957} & \multirow[t]{3}{*}{0.084} \\
\hline GA & $10(21.3)$ & $42(33.6)$ & & \\
\hline GG & $37(78.7)$ & $78(62.4)$ & & \\
\hline \multicolumn{5}{|c|}{ T6000A SNP in IFNG gene } \\
\hline AA & $1(2.1)$ & $9(7.2)$ & \multirow[t]{3}{*}{2.834} & \multirow[t]{3}{*}{0.242} \\
\hline TA & $14(29.8)$ & $46(36.8)$ & & \\
\hline TT & $32(68.1)$ & $70(56)$ & & \\
\hline \multicolumn{5}{|c|}{ T8360C SNP in IFNG gene } \\
\hline $\mathrm{CC}$ & $1(2.1)$ & $5(4)$ & 0.567 & 0.753 \\
\hline
\end{tabular}




\begin{tabular}{|l|l|l|l|l|}
\hline TC & $14(29.8)$ & $41(32.8)$ & & \\
\hline
\end{tabular}

\begin{tabular}{|l|l|l|l|l|}
\hline TT & $32(68.1)$ & $79(63.2)$ & & \\
\hline
\end{tabular}

Table 11. SLC11A1 and IFNG gene polymorphisms in patients with TB, in relation to clinical forms of pulmonary TB, n (\%)

\begin{tabular}{|c|c|c|c|c|c|c|}
\hline Genotype & Infiltrative TB, n=84 & $\begin{array}{l}\text { Focal TB, } \\
n=19\end{array}$ & Disseminated TB, $n=25$ & $\begin{array}{l}\text { Fibrotic cavitary } \\
\text { PTB, } n=11\end{array}$ & $x^{2}$ & 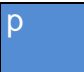 \\
\hline \multicolumn{7}{|c|}{ Asp543Asn SNP in SLC11A1 gene } \\
\hline AA & $1(1.2)$ & $1(5.3)$ & $1(4.0)$ & $1(9.1)$ & \multirow{3}{*}{5.566} & \multirow{3}{*}{0.473} \\
\hline $\mathrm{GA}$ & $22(26.2)$ & $8(42.1)$ & $8(32.0)$ & $4(36.4)$ & & \\
\hline GG & $61(72.6)$ & $10(52.6)$ & $16(64.0)$ & $6(54.5)$ & & \\
\hline \multicolumn{7}{|c|}{ T6000A SNP in IFNG gene } \\
\hline AA & $3(3.6)$ & $3(15.8)$ & $1(4.0)$ & $0(0)$ & \multirow{3}{*}{7.032} & \multirow{3}{*}{0.319} \\
\hline TA & $27(32.1)$ & $5(26.3)$ & $11(44.0)$ & $4(36.4)$ & & \\
\hline TT & 54 (64.3) & $11(57.9)$ & $13(52.0)$ & $7(63.6)$ & & \\
\hline \multicolumn{7}{|c|}{ T8360C SNP in IFNG gene } \\
\hline $\mathrm{CC}$ & $2(2.4)$ & $1(5.3)$ & $1(4.0)$ & $0(0)$ & \multirow{3}{*}{1.339} & \multirow{3}{*}{0.969} \\
\hline TC & $26(31.0)$ & $6(31.6)$ & $9(36.0)$ & $3(27.3)$ & & \\
\hline TT & $56(66.7)$ & $12(63.2)$ & $15(60.0)$ & $8(72.7)$ & & \\
\hline
\end{tabular}

\section{CONCLUSION}

The study findings did not confirm any statistically significant differences between frequencies of SLC11A1 (rs17235409) gene polymorphisms, as well as T6000A IFN- $\gamma$ (rs2430561) and T8360C IFN- $\gamma$ (rs2069718) gene polymorphisms in healthy controls and TB patients. Among Yakut population, the most frequently observed genoptypes were GG for SLC11A1 gene Asp 543 Asn polymorphism, and TT for T6000A IFN- $\gamma$, and T8360C IFN- $\gamma$ polymorphisms.

No association was detected between polymorphisms in genes SLC11A1 (rs17235409) or IFNG (rs2430561, rs2069718) and disease localization, clinical form, culture test result, or clinical progression pattern in patients with TB.

\section{REFERENCES}

[1] M.V. Pavlova, Probl. Tuberk. 11 (2005) 30-33.

[2] V.P. Puzyrev, Med. genet. 1(1) (2002) 44-46.

[3] I.G. Udina, Probl. Tuberk. (2007) 7 27-29.

[4] V.P. Chukanova, L.E. Pospelov, A.F. Malenko, Probl. Tuberk. 7 (2001) 33-36.

[5] A. Ansari, Z. Hasan, G. Dawood, PLoS ONE 6 (2011) 1-5.

[6] J.E. Baghdadi, A.V. Grant, A. Sabri, Pathol. Biol. 61(1) (2013) 11-16.

[7] M. Cellier, G. Govoni, S. Vidal, J. Exp. Med. 180 (1994) 1741-1752.

[8] M. Hashemi, B. Sharifi-Mood, Prague Medical Report 112(1) (2011) 38-43.

[9] M. Shibasaki, T. Yagi, H. Yatsuya, J. Infect. 58(6) (2009) 467-469. DOI: 10.1016/j.jinf.2009.04.002
[10] H.W. Tso, W.K. Ip, W.P. Chong, Genes Immun., 124 (2005) 358-363.

[11] C.R. Vallinoto, S. Ednelza, S. Mauro, Human Immunol. 71 (2010) 692-696. 